\title{
JORDAN LEFT DERIVATIONS AND SOME LEFT DERIVABLE MAPS
}

\author{
JIANKUI LI AND JIREN ZHOU
}

Abstract. Let $\mathscr{A}$ be an algebra and $\mathscr{M}$ be a left $\mathscr{A}$-module. We say that a linear mapping $\varphi: \mathscr{A} \rightarrow \mathscr{M}$ is a left derivable mapping at $P$ if $\varphi(S T)=S \varphi(T)+T \varphi(S)$ for any $S, T \in \mathscr{A}$ with $S T=P$. In this paper, we show that Jordan left derivations or left derivable mappings at zero or unit on some algebras are zero under certain conditions.

Mathematics subject classification (2000): 47B47, 47L35. unit.

Keywords and phrases: Left derivation, Jordan left derivation, left derivable mapping at zero point or

\section{REFERENCES}

[1] M. Ashraf And N. Rehamn, On Lie ideals and Jordan left dervations of prime rings, Arch. Math., 36 (2000), 201-206.

[2] M. Ashraf, N. Rehamn And S. Ali, On Jordan left derivations of Lie ideals in prime rings, Southeast Asian Bulletin of Mathematics, 25 (2001), 379-382.

[3] M. Bresar And J. Vukman, On left derivations and related mappings, Proc. Amer. Math. Soc., 110 (1990), 7-16.

[4] D. Hadwin And J. Li, Local derivations and local automorphisms, J. Math. Anal. Appl., 290 (2004), $702-714$.

[5] D. Hadwin And J. Li, Local derivations and local automorphisms on some algebras, J. Operator Theory, 60 (2008), 29-44.

[6] K. Jun And B. Kim, A note on Jordan left derivations, Bull. Korean Math. Soc., 8 (1996), $221-228$.

[7] M. LAmbrou, On the rank of operators in reflexive algebras, Linear Algebra Appl., 142 (1990), 211-235.

[8] C. Laurie And W. LongstafF, A note on rank one operators in reflexive algebras, Proc. Amer. Math. Soc., 89 (1983), 293-297.

[9] W. LongstafF, Strongly reflexive lattices, J. London Math. Soc., 11 (1975), 491-498.

[10] F. LU AND P. LI, Algebraic isomorphisms and Jordan derivations of $\mathscr{J}$-subspace lattice algebras, Studia Math., 158 (2003), 287-301.

[11] L. Marcoux AND A. Sourour, Conjuagation-invariant subspaces and Lie ideals in nonselfadjioint operator algebras, J. London Math. Soc., 65 (2002), 493-512.

[12] J. VuKMAn, Jordan left derivations on semiprime rings, Math. J. Okayama Univ., 39 (1997), 1-6. 